\title{
Concepciones y prácticas de evaluación en la formación de maestros de educación infantil
}

\author{
Ángela María Gómez-Martínez ${ }^{1}$ \\ Universidad Jorge Tadeo Lozano \\ gomezangela453@gmail.com \\ Angie Yuliana Parra-Mendoza² \\ Universidad San Buenaventura \\ angieyulianaparra03@gmail.com \\ Margie Lorena Soto-Juya ${ }^{3}$ \\ Universidad San Buenaventura \\ margiesoto016@gmail.com
}

DOI: https://doi.org/10.21158/2357514x.v9.n1.2021.3018

Cómo citar este artículo: Gómez-Martínez, A. M.; Parra-Mendoza, A. Y.; Soto-Juya, M. L. (2021). Concepciones y prácticas de evaluación en la formación de maestros de educación infantil. Revista Virtu@lmente, 9(1), Páginas. DOI: https://doi.org/10.21158/2357514x.v9.n1.2021.3018

Fecha de recepción: 02 de octubre de 2020

Fecha de aprobación: 26 de julio de 2021

\section{Resumen}

La investigación tuvo como objetivo caracterizar las concepciones y prácticas de evaluación en la formación de maestros en educación inicial de los programas de Educación Infantil y Educación para la Primera Infancia de la Universidad Pedagógica Nacional, Distrital Francisco José de Caldas y San Buenaventura sede Bogotá. Una vez establecidas las categorías, las concepciones y las prácticas evaluativas, surge la necesidad de analizar si las concepciones que tienen tanto los maestros como los estudiantes de los programas seleccionados guardan relación con las prácticas evaluativas que se desarrollan dentro del aula, ya que el componente teórico es claro, sin embargo, no siempre coherente con su aplicación en la práctica pedagógica. Se orienta esta investigación a partir del diseño metodológico de análisis de contenido, ubicado en el paradigma cualitativo. Así, fue posible identificar la falta de coherencia entre la teoría que los orientadores predican y las prácticas evaluativas que llevan a cabo con los maestros en formación, puesto que el maestro, aunque se basa en diferentes autores y sus teorías, desarrolla sus prácticas de acuerdo con su experiencia. Se puede concluir que, aunque el concepto de evaluación y las prácticas evaluativas se encuentran estrictamente ligadas, si no es clara la concepción que se tiene acerca de la evaluación, muy difícilmente se podrán desarrollar prácticas evaluativas que favorezcan el aprendizaje de los estudiantes, en este sentido, es el maestro quien desde su conocimiento y formación decide sobre la implementación de innovaciones en el aula.

Palabras clave: enseñanza a maestros; maestros en formación; maestros en educación inicial; prácticas de evaluación; práctica pedagógica.

\footnotetext{
${ }^{1}$ Licenciada en Educación Infantil - Universidad San Buenaventura. Estudiante de Artes Plásticas - Universidad Jorge Tadeo Lozano. ORCID: https://orcid.org/0000-0003-3083-0921

${ }^{2}$ Licenciada en Educación para la Primera Infancia - Universidad San Buenaventura. ORCID: https://orcid.org/0000-0003-1493-8588

${ }^{3}$ Licenciada en Educación Infantil - Universidad San Buenaventura. ORCID: https://orcid.org/0000-0002-0073-2522
} 


\title{
Conceptions and practices of the assessment in early childhood teacher education
}

\begin{abstract}
The aim of this research was to characterize the conceptions and assessment practices in the training of early childhood education teachers in the programs of Early Childhood Education and Early Infancy Education at Universidad Pedagógica Nacional, Universidad Distrital Francisco José de Caldas, and Universidad San Buenaventura, Bogotá. Once the categories, conceptions, and assessment practices were established, the need to analyze whether the conceptions held by both teachers and students of the selected programs are related to the assessment practices developed in the classroom arises, since the theoretical component is clear, however, not always consistent with its application in the pedagogical practice. This research is based on the methodological design of content analysis in the qualitative paradigm. Thus, it was possible to identify the lack of coherence between the theory that the counselors preach and the assessment practices that they carry out with the teachers in training, since teachers, although based on different authors and their theories, develop their practices according to their experiences. We might conclude that, although the concept of evaluation and assessment practices are strictly linked, if the concept of evaluation is not clear, it will be very difficult to develop assessment practices that favor student learning. In this sense, it is the teacher who, from his knowledge and training, decides on the implementation of innovations in the classroom.
\end{abstract}

Keywords: teacher education; teachers in training; early childhood education teachers; assessment practices; pedagogical practice.

Concepções e práticas avaliativas

na formação de professores de educação infantil

\section{Resumo}

O objetivo da pesquisa foi caracterizar as concepções e práticas de avaliação na formação de professores em educação básica dos programas de Educação Infantil e Educação na Primeira Infância das Universidades Pedagógica Nacional, Distrital Francisco José de Caldas e San Buenaventura sede de Bogotá. Uma vez estabelecidas as categorias, concepções e práticas avaliativas, surge a necessidade de analisar se as concepções que tanto os professores como os alunos dos cursos selecionados têm se relacionam com as práticas avaliativas que se desenvolvem em sala de aula, uma vez que o componente teórico é claro, no entanto, nem sempre coerente com sua aplicação na prática pedagógica. Esta pesquisa está orientada a partir do desenho metodológico de análise de conteúdo, situado no paradigma qualitativo. Assim, foi possível identificar a falta de coerência entre a teoria que os orientadores pregam e as práticas avaliativas que realizam com os professores em formação, uma vez que o professor, embora baseie-se em diferentes autores e suas teorias, desenvolve suas práticas de acordo com a sua experiência. Pode-se concluir que, embora o conceito de avaliação e as práticas avaliativas estejam estreitamente vinculados, se a concepção de avaliação não for clara, dificilmente desenvolverão práticas avaliativas que favoreçam a aprendizagem do aluno, neste sentido, é o professor que, com base em seu conhecimento e formação, decide sobre a implementação de inovações em sala de aula.

Palavras-chave: ensino a profesores; professores em formaçã; professores em educação básica; práticas de avaliação; prática pedagógica. 
Conceptions et pratiques d'évaluation

de la formation des enseignants de la petite enfance

\begin{abstract}
Résumé
L'objectif de cette recherche vise à caractériser les conceptions et les pratiques d'évaluation de la formation des enseignants des programmes d'éducation à la petite enfance des universités Pédagogique Nationale, Francisco José de Caldas et San Buenaventura de la ville de Bogotá. Une fois établies les catégories, les conceptions et les modalités d'évaluation, nous analyserons la formation des enseignants et étudiants des programmes sélectionnés et les modalités d'évaluation mises en place au sein des classes, dans la mesure où les composantes théoriques sont claires mais pas nécessairement compatibles avec leur application pratique en pédagogie. Cette étude s'oriente vers la conception méthodologique de l'analyse de contenus qualitatifs. Il a ainsi été possible d'identifier le manque de cohérence entre la théorie initiale et les pratiques évaluatives menaient par les enseignants en formation. Enfin, même si le concept d'évaluation et de pratiques évaluatives sont étroitement liés, si la conception de l'évaluation n'est pas claire, il devient extrêmement difficile de développer des pratiques évaluatives améliorant l'apprentissage des étudiants car il revient, in fine, à l'enseignant, de décider, sur la base de ses connaissances et de sa formation, de la mise en place desdites innovations dans la salle de classe.
\end{abstract}

Mots-clés: enseignement enseignant; enseignants en formation; enseignants en formation initiale „; modalités d'évaluation ; pratique pédagogique.

\title{
1. Introducción
}

Por medio de este artículo se pretende dar a conocer los hallazgos obtenidos a partir del desarrollo investigativo por parte de los maestros en formación del Programa de Licenciatura en Educación Para la Primera Infancia de la Universidad de San Buenaventura, sede Bogotá, pertenecientes al Semillero AEI (Aprendiendo a Evaluar la Infancia). Este proceso investigativo se orientó desde el diseño metodológico Análisis de Contenido de Alexander Ruiz Silva (2004), a partir de sus fases: 1) búsqueda: estudio de diferentes documentos teóricos, 2) definición de documentos pertinentes para la investigación, lectura de pénsum y programas analíticos, 3) realización de entrevistas, 4) análisis de concepciones y prácticas evaluativas, 5) identificación de convergencias y divergencias. 
Esta investigación tuvo como objetivo caracterizar las concepciones y prácticas que se tienen de evaluación en la formación de maestros en educación inicial de los programas de Educación Infantil y Educación para la Primera Infancia de la Universidad Pedagógica Nacional, Distrital Francisco José de Caldas y San Buenaventura sede Bogotá.

Una vez establecidas las categorías, las concepciones y las prácticas evaluativas, surge la necesidad de analizar si las concepciones que tienen tanto los maestros como los estudiantes de los programas seleccionados guardan relación con las prácticas evaluativas que se desarrollan dentro del aula, ya que el componente teórico es claro, sin embargo, no siempre coherente con su aplicación en la práctica pedagógica. Esta afirmación concuerda con lo planteado por Argyris (1993) quien explica:

la necesidad de diferenciar las «teorías en uso» de las «teorías proclamadas», entendiendo que las teorías en uso han sido adquiridas a lo largo de la historia personal y profesional [...] y también por muchos mitos y errores pedagógicos, que contribuyen a fosilizar nuestra forma de actuar. (Citado por Pozo, 2014).

Resulta importante comprender la coherencia que debe existir entre la teoría evaluativa que se enseña a las maestras en formación y las prácticas evaluativas que emplean los diferentes profesores de las instituciones universitarias seleccionadas, ya que «teorizar la práctica debería implicar la reflexión del docente sobre su propia práctica, sobre su propia forma de actuar, a la luz de las experiencias educativas más relevantes y de los resultados de la investigación educativa más consistentes» (Pérez-Gómez, Serván-Núñez y SotoGómez, 2015).

Por último, debe ser importante que el maestro tenga claro el concepto de evaluación formativa y lo aplique como lo señalan Díaz y Barriga (2002), con una finalidad «estrictamente pedagógica; regular el proceso de enseñanza-aprendizaje para adaptar o ajustar las condiciones pedagógicas - estrategias, actividades - en servicio de aprendizaje 
de los alumnos» (p. 406). Cabe resaltar que este tipo de evaluación rescata los errores como una oportunidad para aprender y mejorar aquello que hace falta fortalecer, al situar los aprendizajes adquiridos como el reflejo de lo que el estudiante ha logrado y evaluar continuamente su proceso de formación con el fin de reconocer las dificultades que se pueden presentar y así estar en capacidad de brindar una solución de manera oportuna que permita superarlas.

\section{Planteamiento del problema}

Durante el proceso llevado a cabo en el programa de Licenciatura en Educación para la Primera Infancia, y desde lo estudiado en el semillero de investigación AEl, se ha observado la evaluación desde tres perspectivas; en primer lugar, a partir de los cursos que brinda el programa, en los cuales se hace referencia a la evaluación como «formativa» y aseguradora de aprendizajes, tal como lo menciona Álvarez-Méndez (2001): «La evaluación actúa entonces al servicio del conocimiento y del aprendizaje, y al servicio de los intereses formativos a los que esencialmente debe servir» (p. 60). De esta manera, se abordan la importancia tanto de que existan intereses formativos en el momento de evaluar como la oportunidad de corregir los errores cometidos y aprender de ellos, con el fin de crear conocimientos duraderos.

A pesar de ser este el objetivo de la evaluación que se les enseña a los maestros en formación, se genera un quiebre entre la relación teoría-acción, ya que algunos orientadores emplean la evaluación no como un medio para asegurar aprendizajes, sino para calificar o descalificar las habilidades de sus estudiantes. 
Esta creencia, por la cual se afirma que la evaluación se encuentra directamente relacionada con un valor numérico, que se le asigna a una prueba elaborada por un estudiante, va en contra de lo que se plantea Álvarez-Méndez (2001, p. 11),

evaluar tampoco es clasificar ni es examinar ni aplicar tests. Paradójicamente, la evaluación tiene que ver con actividades de calificar, medir, corregir, clasificar, certificar, examinar, pasar test, pero no se confunde con ellas. Comparten un campo semántico, pero se diferencian por los recursos que utilizan y los usos y fines a los que sirve. De estas actividades artificiales no se aprende. Respecto a ellas la evaluación trasciende. Justo donde ellas no alcanzan empieza la evaluación educativa.

Surge, entonces, la necesidad de reconocer de qué manera se enseña a los maestros a evaluar, puesto que más adelante puede ser el reflejo de las prácticas evaluativas que ellos van a ejecutar en el aula con sus estudiantes. Para dar respuesta a estos interrogantes se encontró que mediante el curso de «evaluación formativa», ofertado por la Universidad de San Buenaventura a los maestros en formación, se busca fortalecer la comprensión del concepto de evaluación, con el fin de crear conciencia acerca de las prácticas evaluativas ejercerán en su vida profesional. Tal como lo menciona Grau-Company y Gómez-Lucas (2005), la evaluación es parte fundamental del aprendizaje en cuanto no solo constata los cambios en los estudiantes, sino que permite evidenciar la eficacia de los métodos y recursos, los programas y demás factores, para facilitar la toma de decisiones orientadas a reconducir el proceso.

Evidenciado lo anterior, surge la segunda perspectiva que hace referencia a la inquietud sobre cómo se está enseñando a los estudiantes de las universidades San Buenaventura, Distrital Francisco José de Caldas y Pedagógica, con énfasis en las facultades de educación, y si este concepto que se plantea es coherente con las prácticas evaluativas que los profesores de los diferentes programas utilizan para evaluar sus aprendizajes. Lo anterior 
en razón a que, como sugiere Rosales (2014), la evaluación puede estar sujeta a la conceptualización e interpretación de cada docente, en procura de hacer uso de instrumentos que cumplan con los criterios de calidad que respondan a sus necesidades.

Por tanto, los procesos de evaluación que se utilizan con los estudiantes deben ser coherentes con los discursos empleados por los orientadores de los diferentes cursos brindados en cada una de las universidades, pues es incoherente que en las instituciones universitarias donde los maestros en formación hacen inmersión se utilice aún el método evaluativo como una amenaza o un castigo hacia los estudiantes; pues esto podría afectar su proceso de aprendizaje, aun cuando en realidad el valor de la evaluación radica en valorar, apreciar, estimar el valor de las cosas inmateriales.

Es por todo esto que se desglosa la tercera y última perspectiva, la cual hace referencia a la manera en que los maestros egresados de las universidades evalúan a los niños, pues la aplicación que le otorguen a la práctica evaluativa en el aula puede ser un reflejo de los procesos evaluativos que recibieron en su formación como maestros, ya que según, Álvarez-Méndez (2001, p. 12),

debe entenderse la evaluación como actividad crítica de aprendizaje, porque se asume que la evaluación es aprendizaje en el sentido que por ella adquirimos conocimiento. El profesor aprende para conocer y para mejorar la práctica docente en su complejidad, y para colaborar en el aprendizaje del alumnado conociendo las dificultades que tienen que superar, el modo de resolverlas y las estrategias que pone en funcionamiento. El alumno aprende de y partir de la propia evaluación y de la corrección, de la información contrastada que le ofrece el profesorado, que será siempre crítica y argumentada, pero nunca descalificadora ni penalizadora. 


\section{Diseño metodológico}

Para dar respuesta a las perspectivas mencionadas, se orienta esta investigación a partir del diseño metodológico análisis de contenido, ubicado en el paradigma cualitativo, el cual, al tener una dinámica dentro de la investigación social, no solo responde a los objetivos de los investigadores, sino, además, a las necesidades de los contextos. Como lo menciona Ruiz-Silva (2004), «los procedimientos seguidos en su utilización nos permiten comprender la complejidad de la realidad social que estamos interesados en estudiar, en lugar de simplificarla y reducirla a mínimos esquemas de representación» ( $p$. 45). Por tanto, se escoge este tipo de investigación, pues permite recoger las voces de las personas involucradas, en este caso en el contexto universitario, y junto con ello observar la complejidad de su realidad social; de igual forma, es de tipo cualitativo, ya que permite analizar directamente las experiencias relatadas por cada uno de los actores.

Inicialmente, se realizó la búsqueda y lectura de diferentes referentes teóricos con el fin de dar sustento a la fase del desarrollo, en la cual se seleccionaron aquellos que realmente eran pertinentes para la investigación. Con relación a este proceso fue necesario realizar una lectura de los documentos base de los programas de educación infantil seleccionados y sus planes de estudio, revisar los proyectos realizados en las universidades que guardaban relación con el tema a investigar, al igual que textos escritos por expertos en el tema de la evaluación.

A partir de este proceso fue posible identificar que una de las tres universidades que se tuvieron en cuenta para la realización de este proyecto no contaba con un curso relacionado con el tema de evaluación, de manera que se cuestiona el papel que cumple la evaluación en los procesos de formación de los maestros en educación infantil. 
Dando continuidad al proceso de investigación se dio paso a la tercera fase. Esta se refiere a la realización de entrevistas, para lo cual se aplicaron entrevistas semiestructuradas a 38 maestros en formación. Una vez recolectados y transcritos los testimonios de los entrevistados de las diferentes universidades, se dio paso a la cuarta fase del desarrollo de la investigación, que busca el análisis de las categorías de estudio «concepciones» y «prácticas evaluativas».

Las categorías de estudio nombradas fueron fundamentales para analizar y comprender las concepciones que interiorizan los profesores y cómo estos ejecutan prácticas evaluativas con sus estudiantes de acuerdo con el ideal de evaluación que se ha construido a lo largo del tiempo.

Una vez desarrollado el proceso de categorización se dio paso al nivel de superficie del análisis de contenido. A fin de desarrollarlo se realizó una lectura minuciosa de las entrevistas con el propósito de obtener los elementos más relevantes del discurso dado por los maestros en formación que fueron entrevistados, teniendo en cuenta las categorías mencionadas. Posteriormente, esta información se ubicó en matrices que tenían como objetivo organizar segmentos textuales del discurso de los maestros en formación para facilitar su análisis.

Obtenida la información en el nivel de superficie, se analizó detenidamente con el fin de definir convergencias y divergencias de los discursos de cada uno de los entrevistados, comenzando entonces el desarrollo del nivel analítico de la investigación, para así lograr analizar las concepciones y prácticas que se tienen de evaluación en la formación de maestras de distintas instituciones universitarias. Una vez agrupadas las convergencias y divergencias en el nivel analítico, se pasa a la sexta fase de la investigación, la cual se basó 
en dotar de sentido la información clasificada en las fases anteriores, teniendo en cuenta el conocimiento por parte de las investigadoras y lo que arrojaron las evidencias.

A partir de este proceso de análisis de la información fue posible evidenciar que las prácticas evaluativas se dividen en dos posturas. Una direccionada por los maestros hacia lo tradicional, entendidas desde Bustamante-Zamudio y Díaz-Monroy (2003) como un ejercicio que permite evidenciar un resultado en periodos determinados, ya que se resalta la importancia que tiene la nota o calificación para determinar el proceso académico del estudiante mediante métodos que permiten únicamente valorar de una manera cuantitativa los conocimientos adquiridos por los estudiantes, como lo son las pruebas estandarizadas. Esta afirmación se relaciona con lo siguiente: «Sé que muchas maestras no lo hacen, incluso en la universidad muchos maestros no nos evalúan un proceso sino nos evalúan por cortes para medir no tu proceso sino como cortar algo y hasta aquí evaluaste, y número, hasta qué punto y ya, o sea tu proceso no me importa» (Laura Fajardo, comunicación personal, 18 de noviembre de 2019).

Por esta razón, se hace énfasis en el aprendizaje netamente memorístico, reflejado a partir de la implementación de herramientas tales como parciales, trabajos escritos, cuestionarios, entre otros, que se encuentran relacionadas con el currículo que establece la institución, puesto que estas prácticas están ligadas a la concepción administrativa que los entes gubernamentales imponen en el país.

Por otra parte, la segunda postura se ve direccionada de manera formativa, ya que algunos estudiantes realizan sus prácticas evaluativas como un proceso reflexivo, tal como lo menciona el entrevistado (Juan Flores, comunicación personal, 18 de noviembre de 2019). 
Son más llamativos los espacios en donde estamos llamados a la reflexión desde la autonomía, ¿no? No solamente porque uno se ponga la nota, porque finalmente siempre se ha hecho énfasis en eso, la nota no importa y la nota no lo define a usted como estudiante, sino los procesos en los que uno se puede auto reflexionar y los procesos donde uno puede criticarse a uno mismo y saber, bueno, qué fue lo que realmente me quedó de esta clase, para qué me sirve esto como maestro o maestra en formación, son los espacios en los que uno más aprende.

Es de esta manera que se permite reconocer las capacidades y dificultades de los niños, utilizando herramientas diferentes a las tradicionales, como, por ejemplo, proyectos que estimulen el trabajo en equipo, infografías, realización de organizadores gráficos, debates en los que los maestros en formación se sientan libres de expresar sus opiniones, se resalte la voz de los alumnos y se logren evidenciar las fortalezas y debilidades que tienen respecto al tema que se esté abordando.

Tal como lo menciona Álvarez-Méndez, (2001) «el diálogo y la conversación son medios de conocimiento y de evaluación que no se utilizan con la frecuencia que corresponde a un recurso natural» (p. 56). Al igual el feedback, el cual le permite al maestro en formación darse cuenta de qué alcances ha tenido y qué hace falta mejorar de su proceso, de manera que le da al aprendizaje la importancia pertinente y permite tanto al orientador como al maestro en formación aprender de dicha experiencia. Por tanto, «el feedback es un canal personal de comunicación con los alumnos sobre algo en lo que estos suelen invertir una considerable cantidad de tiempo y de esfuerzo. Al aprendiz le importa su trabajo y como este será juzgado» (Boud y Molloy, 2015, p. 13).

Finalmente, para algunos es relevante que las prácticas evaluativas contemplen otras alternativas de enseñanza como lo es el juego, el cual se emplea como una estrategia dinámica y flexible de aprendizaje y evaluación que permite la interacción entre las partes 
de manera fácil y divertida, tal como lo explica Bustamante-Zamudio y Díaz-Monroy (2003).

Es importante también que en el momento de evaluar se implementen herramientas que permitan documentar el proceso de cada niño, lo que da valor a las experiencias propias de cada uno de ellos, al igual que involucrar a las familias y al estudiante en la construcción de la práctica evaluativa, de modo que eduquen respecto a lo que significa dicho concepto, la manera en la que el maestro va a evaluar y los objetivos que este se ha planteado para darle desarrollo a esta. Con relación a lo anterior se destaca lo mencionado por el entrevistado (Daniela Rodríguez, comunicación personal, 18 de noviembre de 2019): «se considera que la evaluación no es un proceso lineal, sino que varía de acuerdo en la manera en que se guía el curso y por supuesto la manera en que los estudiantes se involucran con su proceso de formación».

Por último, fue posible identificar que la falta de coherencia entre la teoría que los orientadores predican y las prácticas evaluativas que llevan a cabo con los maestros en formación es confusa, puesto que el maestro habla basado desde diferentes autores y sus teorías, pero desarrolla sus prácticas de acuerdo con su experiencia.

Es importante mencionar que estas vivencias están, en ocasiones, mediadas por cada uno de los actores que han intervenido en el proceso de formación del maestro, lo cual ayuda a que él cree una concepción de evaluación muchas veces difícil de desligar de su zona de confort, de manera que el maestro orientador teme a innovar en su práctica, ya que resulta más fácil imitar prácticas evaluativas que considera efectivas desde su propia experiencia a intentar alternativas que, por ser nuevas, pueden «no funcionar», planteamientos que se relacionan con lo que menciona Flórez (2000): «Los profesores 
promueven con sus pruebas objetivas, fáciles de diseñar, de aplicar y de corregir, y útiles para evadir las réplicas y quejas de los estudiantes ante las malas notas» (p.179).

\section{Conclusiones}

Para dar conclusión a este escrito, resulta fundamental guardar coherencia entre el discurso que emplea el maestro acerca de la evaluación y las prácticas evaluativas que desarrolla dentro del aula, ya que estas influyen en la construcción que el maestro en formación apropia acerca de qué es evaluar y cómo debe evaluar a sus estudiantes. Puesto que el maestro es el responsable de que estas cobren un sentido formativo, al dar ejemplo a los alumnos.

Inicialmente, en el planteamiento del problema se dieron a conocer tres tensiones de las cuales se puede concluir, primero, la importancia de concebir la evaluación como formativa, de modo que los errores se convierten en oportunidades de aprendizaje para el estudiante. Es por esto que resulta de suma importancia comprender cómo la evaluación trasciende los estándares de calificación, medición y validación de un simple error. Por el contrario, se le otorga un valor a esta como un proceso de reflexión y aprendizaje de forma bilateral, es decir, así como el estudiante puede mejorar gracias a la evaluación, los maestros también pueden fortalecer su quehacer docente gracias a lo que aprenden de ella.

En la segunda perspectiva se logró reconocer que existe una coherencia entre la concepción que tiene la maestra en cuanto a evaluación formativa, ya que a partir de su discurso fundamentó el sentido de la evaluación como una propuesta alternativa en la que no importa el instrumento que se aplique, sino el sentido que se le otorgue a este; es por esto que la evaluación muy difícilmente puede desligarse de la educación. 
Para dar respuesta a la tercera perspectiva, se pudo concluir que sí hay repercusiones en la construcción del concepto de evaluación que ha logrado apropiar el maestro en formación, ya que esto será el reflejo tanto del concepto de evaluación que le enseñaron como de las prácticas evaluativas que aplicaron para que fuese evaluado; por tanto, será difícil que el maestro en formación se desligue de la concepción construida y pase a replicar esto con los niños.

Adicionalmente, se puede concluir que el concepto de evaluación y las prácticas evaluativas se encuentran estrictamente ligadas, ya que si no es clara la concepción que se tiene acerca de la evaluación, muy difícilmente se podrán desarrollar prácticas evaluativas que favorezcan el aprendizaje de los estudiantes. Es el maestro quien desde su conocimiento y formación implementa y decide si innova en el aula.

Finalmente, es importante que el maestro se desligue o deje de refugiarse en que la normatividad es la que «le impone» cómo debe desarrollar la práctica evaluativa en el aula, puesto que es él quien desde su conocimiento y formación implementa y decide si innova en el aula o por el contrario opta por seguir desarrollando prácticas evaluativas tradicionales sin un sentido formativo, en las cuales muchas veces se evalúan contenidos que no han sido enseñados y que, por consiguiente, no se van a reflejar en los aprendizajes de los estudiantes.

Una vez se reconozca la evaluación como un proceso formativo es posible comprender que no se trata del instrumento que se utilice para evaluar sino del sentido que se le otorgue a este en el momento de aplicarlo en el aula, pues es allí donde se puede lograr un cambio en el concepto de evaluación, concibiéndola no como la aplicación de una prueba en la que se validan aprendizajes, sino como una herramienta por medio de la que se puede reconocer el proceso de formación del estudiante. 


\section{Referencias}

Álvarez-Méndez, J. (2011). Evaluar para conocer, examinar para excluir. Madrid: Morata.

Argyris, C. (1993). Knowledge for action: A guide to overcoming barriers to organizational change. San Francisco: Jossey-Bass.

Boud, D., y Molloy, E. (Coords.) (2015). El feedback en educación superior y profesional: comprenderlo y hacerlo bien. Madrid: Narcea.

Bustamante-Zamudio, G; Díaz-Monroy, L. G. (2003). Factores asociables al desempeño de los estudiantes: ilustrado para la evaluación de impacto del plan de universalización en el área de lenguaje. Bogotá: Universidad Nacional de Colombia

Díaz, F; Barriga, A. (2002) Estrategias Docentes para un Aprendizaje Significativo: una Interpretación Constructivista. México: Mc Graw Hill.

Flórez, R. (2000). Evaluación pedagógica y cognición. Bogotá: McGraw-Hill.

Grau-Company, S.; Gómez-Lucas, C. (2005). La evaluación un proceso de cambio para el aprendizaje. En Evaluación de los aprendizajes en el Espacio Europeo de Educación Superior. (17-32). Alcoy: Marfil. Recuperado de https://bit.ly/2ZsrlTQ

Pérez-Gómez, A.; Serván-Núñez, M.; Soto-Gómez, E. (2015). Lesson studies: re-pensar y re-crear el conocimiento práctico en cooperación. Revista Interuniversitaria de Formación del Profesorado, 29(3), 81-101.

Pozo, J.I. (2014). Psicología del Aprendizaje Humano: Adquisición de conocimiento y cambio personal. Madrid: Morata.

Rosales, M. (2014). Proceso evaluativo: evaluación sumativa, evaluación formativa y Assesment su impacto en la educación actual. En Congreso Iberoamericano de Ciencia, Tecnología, Innovación y Educación. Buenos Aires, Argentina, 13-14 de noviembre. Recuperado de https://bit.ly/3m13xhH

Ruiz-Silva, A (2004). Texto, testimonio y metatexto: el análisis de contenido en la investigación en educación. La práctica investigativa en ciencias sociales. En A. Jiménez-Becerra; A. TorresCastillo (Comp.) La práctica investigativa en ciencias sociales. (44-59). Bogotá: Universidad Pedagógica Nacional. Recuperado de https://bit.ly/3kGWZFn 